\title{
Fusion neural networks for plant classification: learning to combine RGB, hyperspectral, and lidar data
}

\author{
Victoria M Scholl ${ }^{\text {Corresp., 1, }}{ }^{2}$, Joseph McGlinchy ${ }^{1}$, Teo Price-Broncucia ${ }^{3}$, Jennifer K Balch ${ }^{1,2}$, Maxwell B Joseph ${ }^{1}$ \\ ${ }^{1}$ Earth Lab, Cooperative Institute for Research in Environmental Science, University of Colorado at Boulder, Boulder, Colorado, United States \\ 2 Department of Geography, University of Colorado at Boulder, Boulder, Colorado, United States \\ 3 Department of Computer Science, University of Colorado at Boulder, Boulder, Colorado, United States \\ Corresponding Author: Victoria M Scholl \\ Email address: Victoria.Scholl@colorado.edu
}

Airborne remote sensing offers unprecedented opportunities to efficiently monitor vegetation, but methods to delineate and classify individual plant species using the collected data are still actively being developed and improved. The Integrating Data science with Trees and Remote Sensing (IDTReeS) plant identification competition openly invited scientists to create and compare individual tree mapping methods. Participants were tasked with training taxon identification algorithms based on two sites, to then transfer their methods to a third unseen site, using field-based plant observations in combination with airborne remote sensing image data products from the National Ecological Observatory Network (NEON). These data were captured by a high resolution digital camera sensitive to red, green, blue (RGB) light, hyperspectral imaging spectrometer spanning the visible to shortwave infrared wavelengths, and lidar systems to capture the spectral and structural properties of vegetation. As participants in the IDTReeS competition, we developed a two-stage deep learning approach to integrate NEON remote sensing data from all three sensors and classify individual plant species and genera. The first stage was a convolutional neural network that generates taxon probabilities from RGB images, and the second stage was a fusion neural network that "learns" how to combine these probabilities with hyperspectral and lidar data. Our two-stage approach leverages the ability of neural networks to flexibly and automatically extract descriptive features from complex image data with high dimensionality. Our method achieved an overall classification accuracy of 0.51 based on the training set, and 0.32 based on the test set which contained data from an unseen site with unknown taxa classes. Although transferability of classification algorithms to unseen sites with unknown species and genus classes proved to be a challenging task, developing methods with openly available NEON data that will be collected in a standardized format for 30 years allows for continual improvements and major gains for members of the computational ecology community. We 
outline promising directions related to data preparation and processing techniques for further investigation, and provide our code to contribute to open reproducible science efforts. 


\title{
Fusion neural networks for plant classification: learning to combine RGB, hyperspectral, and lidar data
}

\author{
Victoria M. Scholl ${ }^{1,2}$, Joseph McGlinchy ${ }^{1}$, Teo Price-Broncucia ${ }^{3}$, Jennifer \\ K. Balch ${ }^{1,2}$, and Maxwell B. Joseph ${ }^{1}$ \\ ${ }^{1}$ Earth Lab, Cooperative Institute for Research in Environmental Science, University of \\ Colorado at Boulder, Colorado, United States \\ ${ }^{2}$ Department of Geography, University of Colorado at Boulder, Colorado, United States \\ ${ }^{3}$ Department of Computer Science, University of Colorado at Boulder, Colorado, United \\ States \\ Corresponding author: \\ Victoria M. Scholl ${ }^{1}$ \\ Email address: victoria.scholl@colorado.edu
}

\begin{abstract}
Airborne remote sensing offers unprecedented opportunities to efficiently monitor vegetation, but methods to delineate and classify individual plant species using the collected data are still actively being developed and improved. The Integrating Data science with Trees and Remote Sensing (IDTReeS) plant identification competition openly invited scientists to create and compare individual tree mapping methods. Participants were tasked with training taxon identification algorithms based on two sites, to then transfer their methods to a third unseen site, using field-based plant observations in combination with airborne remote sensing image data products from the National Ecological Observatory Network (NEON). These data were captured by a high resolution digital camera sensitive to red, green, blue (RGB) light, hyperspectral imaging spectrometer spanning the visible to shortwave infrared wavelengths, and lidar systems to capture the spectral and structural properties of vegetation. As participants in the IDTReeS competition, we developed a two-stage deep learning approach to integrate NEON remote sensing data from all three sensors and classify individual plant species and genera. The first stage was a convolutional neural network that generates taxon probabilities from RGB images, and the second stage was a fusion neural network that "learns" how to combine these probabilities with the hyperspectral and lidar data. Our twostage approach leverages the ability of neural networks to flexibly and automatically extract descriptive features from complex image data with high dimensionality. Our method achieved an overall classification accuracy of 0.51 based on the training set, and 0.32 based on the test set which contained data from an unseen site with unknown taxa classes. Although transferability of classification algorithms to unseen sites with unknown species and genus classes proved to be a challenging task, developing methods with openly available NEON data that will be collected in a standardized format for 30 years allows for continual improvements and major gains for members of the computational ecology community. We outline promising directions related to data preparation and processing techniques for further investigation, and provide our code to contribute to open reproducible science efforts.
\end{abstract}

\section{INTRODUCTION}

Understanding the species composition of individual trees within forests is essential for monitoring biodiversity (Nagendra, 2001; Wang et al., 2010), invasive species (Asner et al., 2008; He et al., 2011), terrestrial carbon (Schimel et al., 2015; Jucker et al., 2017), and disturbance regimes (Kulakowski et al., 2003; Senf et al., 2017). Remote sensing enables us to more efficiently map and monitor vegetation than using traditional field-based methods alone, using platforms ranging in scale from drones to satellites carrying a wide variety of sensors (Kerr and Ostrovsky, 2003; White et al., 2016; Lucash et al., 2018). Different types of passive and active imaging sensors provide unique information about ecosystems that may be most useful when combined (Anderson et al., 2008; Tusa et al., 2020). Multispectral cameras 
are accessible, affordable and typically require minimal post-processing to be ready for analysis (Gini et al., 2018; Abdollahnejad and Panagiotidis, 2020). Hyperspectral data are valuable for their ability to capture spectral signatures beyond the visible wavelengths, which often contain descriptive reflectance characteristics across plant types and conditions (Dalponte et al., 2012; Ballanti et al., 2016). Active sensors such as Light Detection and Ranging (lidar) emit pulses of laser light and record the amount and intensity of reflected energy. Lidar data provide structural information about the height, shape, and variability of tree crowns (Heinzel and Koch, 2011; Koenig and Höfle, 2016).

Any one data source could be used for plant species classification, but combining information from multiple sources is valuable, albeit difficult (Torabzadeh et al., 2014; Anderson et al., 2008; Asner et al., 2012). Deep neural networks automatically extract intricate patterns and identify trends from large volumes of data (LeCun et al., 2015), which makes them useful for classification and data fusion tasks (Zhu et al., 2017; Ma et al., 2019), including plant species classification (Brodrick et al., 2019; Fricker et al., 2019; Zhang et al., 2020; Onishi and Ise, 2021). At a high level, neural networks are flexible function approximators that learn a mapping from inputs (e.g., spectral or lidar data) to outputs (e.g., species classes), by way of a sequence of matrix multiplications and nonlinearities. By providing different kinds of input to the same network (e.g., a multimodal network that ingests spectral and lidar data), neural networks learn how to fuse different data sources, in contrast to more manual approaches in which a human analyst decides how to combine disparate data ahead of time (Diaz et al., 2020).

Here we describe the deep learning classification approach used by the Jeepers Treepers team for the Integrating Data science with Trees and Remote Sensing (IDTreeS) 2020 plant classification challenge (https://idtrees.org). IDTReeS uses publicly available data from the National Ecological Observatory Network (NEON), funded by the National Science Foundation (NSF) to measure long-term ecological change at 81 field sites in 20 ecoclimatic domains across the United States, Alaska, Hawaii and Puerto Rico (Keller et al., 2008). The NEON data provided for this competition include both field-based plant measurements and airborne remote sensing data products derived from high resolution red, green, blue (RGB) digital camera imagery, hyperspectral imagery across the visible to shortwave infrared wavelengths, and light detection and ranging (lidar) data (Johnson et al., 2010). By participating in an open competition, teams are encouraged to innovate and accelerate their computational methods development (Carpenter, 2011). An earlier iteration of this competition used NEON data from a single forest to convert images into information on individual trees (Marconi et al., 2019), while this 2020 competition used data from three sites to compare how transferable teams' methods were to unseen sites. Classifier transferability to out-of-sample spatial, temporal, and geographic regions is particularly important in cases where data are limited (Wu et al., 2006; Moon et al., 2017). In addition to emphasizing method generalization across sites, this competition tasked teams with designing classification models that can deal with species and genera from outside of the training set. We begin with a description of our data processing steps, then segue into a three stage classification pipeline, and finally report our results along with ideas for future investigation. All of the data and processing tools that we used are open source and publicly available to support and enable reproducible science.

\section{METHODS}

\section{Study area}

The IDTReeS competition included data from 3 different NEON domains in the southeastern United States, each with distinct ecological and climatic characteristics (Fig. 1). Ordway-Swisher Biological Station (OSBS) in Florida in the Southeast NEON domain features a mixed forest of hardwood and conifers, mostly dominated by pines. Mountain Lake Biological Station (MLBS) in Virginia in the Appalachians and Cumberland Plateau NEON domain is mainly composed of hardwood trees. Talladega National Forest (TALL) in Alabama in the Ozarks complex NEON domain is dominated by mixed hardwoods and conifers (mostly pine), with a tree species composition that is largely a mixture of species found in OSBS and MLBS. Training data for the competition were provided at two of the three NEON sites, MLBS and OSBS, and then our classification method was evaluated at those two sites in addition to the TALL site (where our classifier has not seen data).

\section{Data processing}

The IDTReeS research team provided publicly available geospatial and tabular data for use in this competition (Graves and Marconi, 2020). We processed raw NEON data to generate a feature vector for 


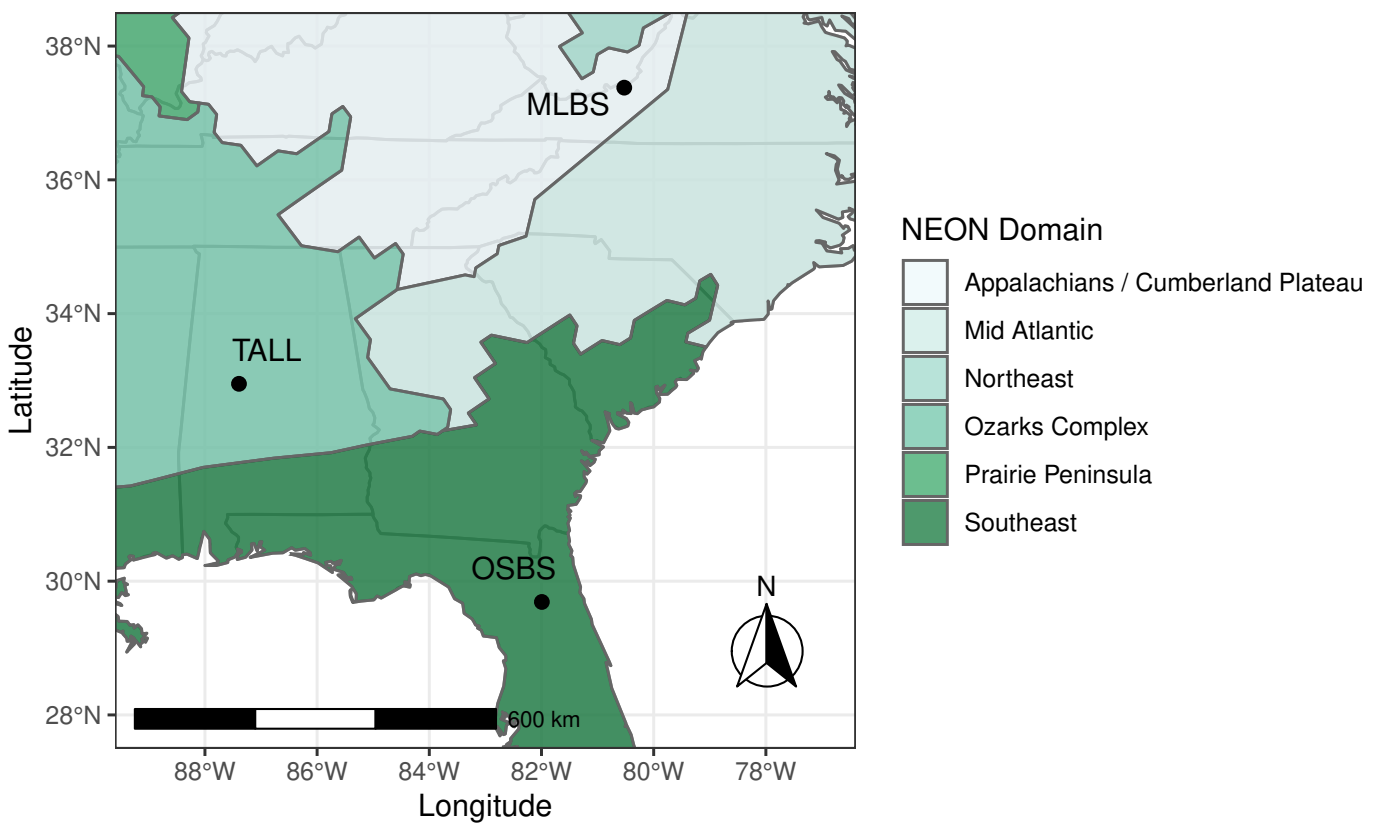

Figure 1. Study Area. The data provided for the tree mapping competition belong to three National Ecological Observatory Network (NEON) sites: Ordway-Swisher Biological Station (OSBS) in Florida, Mountain Lake Biological Station (MLBS) in Virginia, and Talladega National Forest (TALL) in Alabama. The sites span three separate NEON domains in the southeastern United States, each with distinct ecological and climatic characteristics, although the TALL site has a species composition that is largely a mixture of species found in OSBS and MLBS.

each individual plant canopy, then passed these vectors to a multimodal neural network (Ngiam et al., 2011) that ultimately makes the taxon predictions (Fig. 2). The raw geospatial data that we used include high resolution orthorectified red, green, blue (RGB) digital camera imagery with $10 \mathrm{~cm}$ spatial resolution (NEON.DP1.30010), hyperspectral reflectance data from the NEON Imaging Spectrometer with $1 \mathrm{~m}$ resolution (NEON.DP3.30006), and discrete lidar point cloud data with a point density of $\approx 3.15$ points per square meter (NEON.DP1.30003) provided by the IDTreeS competition at each of the NEON ground plots (Graves and Marconi, 2020; NEON, 2020).

These data products are derived from both active and passive remote sensing systems onboard the NEON Airborne Observation Platform (AOP) to capture the structural and spectral characteristics of vegetation (Kampe et al., 2010a). The high resolution RGB images are collected by an Optech D8900 digital color camera and capture fine spatial details of the tree crowns across the visible wavelengths (Gallery et al., 2015). The hyperspectral reflectance data have 426 spectral bands spanning the visible to infrared regions from 380-2510nm in increments of $5 \mathrm{~nm}$ (Karpowicz and Kampe, 2015). The lidar data points representing the $\mathrm{x}, \mathrm{y}, \mathrm{z}$ location of surface features and the ground in three-domensional space were acquired by the Optech Incorporated Airborne Laser Terrain Mapper Gemini instrument with a near infrared laser that operates at 1064nm (Krause and Goulden, 2015). The NEON AOP flies at typical altitude of $1000 \mathrm{~m}$ above ground level and is intended to collect airborne data at each NEON site's peak phenological greenness. These geospatial data products were provided in $20 \mathrm{~m} \times 20 \mathrm{~m}$ tiles representing the size of individual sampling plots.

Woody plant vegetation structure field data (NEON.DP1.10098) in tabular form collected based on NEON's Terrestial Observation System protocol (Thorpe et al., 2016) were provided as well, contributing information on individual tree identifiers, sampling locations, and taxonomic species or genera labels. Individual tree crown delineations were generated and provided by the IDTReeS competition research 
group for the classification task. Each canopy polygon was a rectangular bounding box that represents the maximum crown extent for each individual tree. Each canopy polygon was associated with a record in the NEON field data. We extracted data independently for each mapped tree canopy. First we generated a rectangular RGB image subset for each individual plant by using the provided canopy polygons to crop the RGB image tiles. Then we extracted hyperspectral reflectance data from the spatial centroid pixel within each canopy polygon. Finally, we generated pseudo-waveforms from lidar data by computing the density of point cloud returns within the boundary of each canopy polygon using 39 vertical bins of height. We split the provided data randomly into a training $(75 \%)$ and initial validation $(25 \%)$ set so that each individual tree was associated with just one of the data partitions. Note that we used this initial validation set to help tune our RGB classification step in the first stage of our approach. For the final evaluation of our classification method, we were provided with an independent set of data without taxa labels.

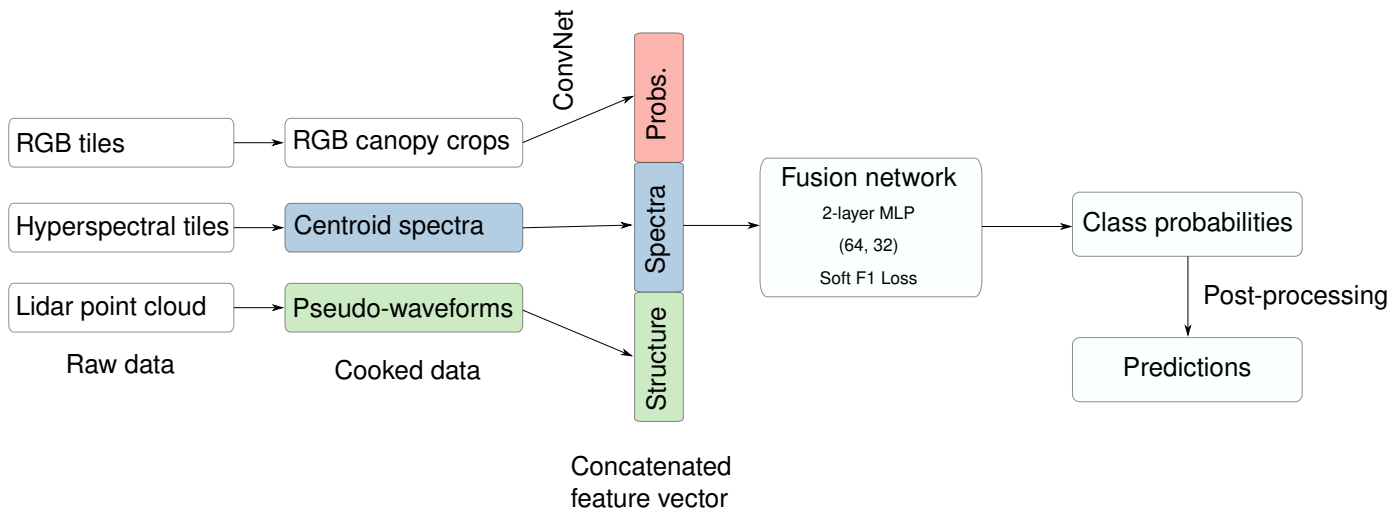

Figure 2. The workflow. We processed raw remote sensing data products into formats that describe the spectral and structural characteristics for each individual plant canopy. We used a pre-trained Convolutional Neural Network (ConvNet) to estimate taxon probabilities using the red, green, blue (RGB) cropped canopy images, and combined these taxon probabilities with hyperspectral reflectance spectra and lidar-derived pseudo-waveforms into a concatenated feature vector. This feature vector was the input to the so-called "fusion network", a 2-layer multilayer perceptron (MLP) with two hidden layers (size 64 and 32) and trained using a custom "soft F1" loss function, to predict taxon class probabilities for each individual plant. We then applied post-processing including a threshold to assign individuals to an "other" class when the classification confidence was low. Finally, we produced predictions of taxon probabilities.

\section{Feature extraction from RGB data}

We used the cropped rectangular RGB canopy images as input to fine-tune a convolutional neural network (CNN) pretrained on the ImageNet dataset (Deng et al., 2009). CNNs have been shown effective in classification of high resolution remote sensing images by learning textural and spatial relationships through many stages of convolutional filters and pooling layers (Zhu et al., 2017).

We split the individual tree RGB canopy images randomly into training (80\%) and validation (20\%) subsets to tune the CNN. The RGB data consisted of 1052 individuals each belonging to one of 31 taxa (Table 1). There was notable class imbalance; approximately one-third of the trees were PIPA2 (Pinus palustris, longleaf pine) while many species or genera only had one or two samples represented in the data set.

The size and dimensions of the rectangular canopy polygons were quite variable (Fig. 3). Since the pretrained CNN requires each image to have the same dimensions, we transformed each rectangular RGB canopy cropped image to be $224 \times 224$ pixels using a combination of cropping and resizing, and each image was normalized based on the mean and standard deviation of the ImageNet data set. We labeled each of the resized and normalized RGB canopy images with its respective taxon identification code (Fig. 4).

\section{ResNet evaluation}

We tested a series of ResNet CNNs (He et al., 2016) to generate a probability for each taxon class from the RGB image chips. We loaded pretrained weights generated from the ImageNet dataset, using ResNets 

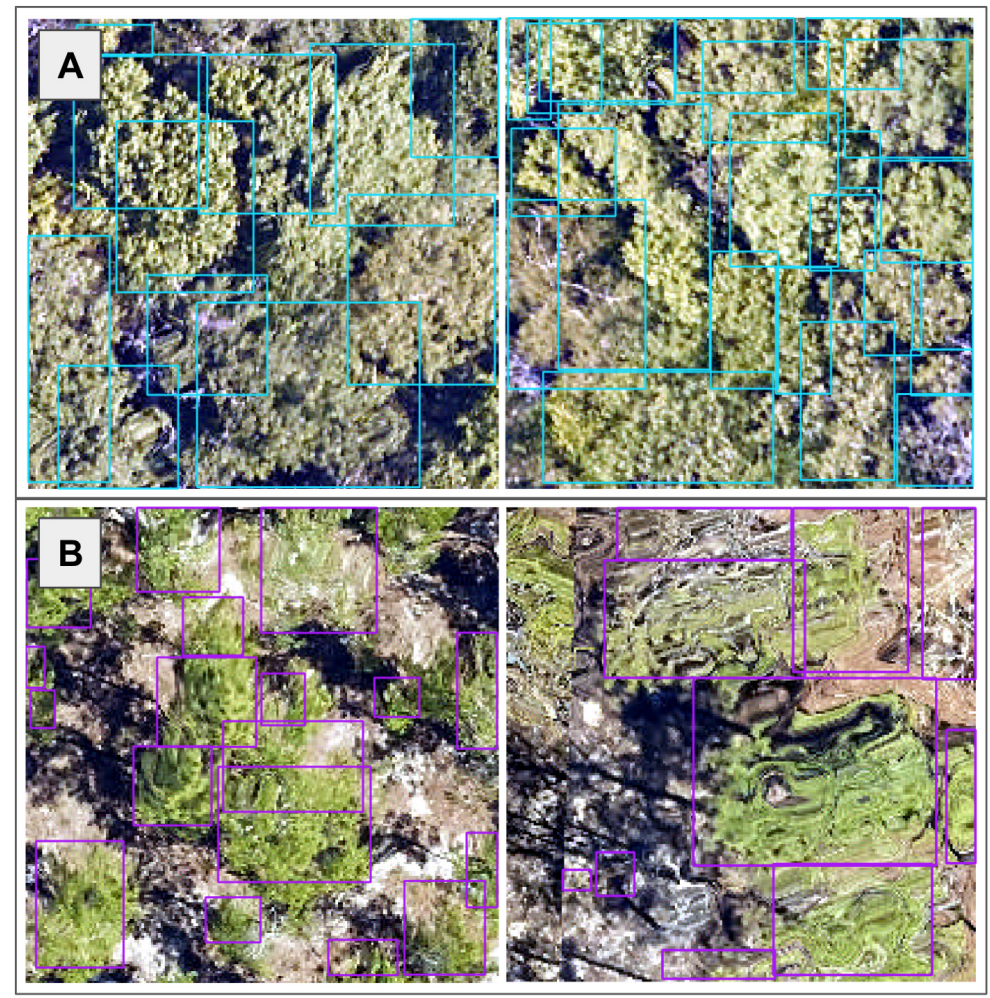

Figure 3. We used the individual tree crown rectangular polygons to clip remote sensing image layers, such as the $10 \mathrm{~cm}$ high spatial resolution red, green, blue (RGB) data shown here at the (A) Ordway-Swisher Biological Station (OSBS) and (B) Mountain Lake Biological Station (MLBS) sites.

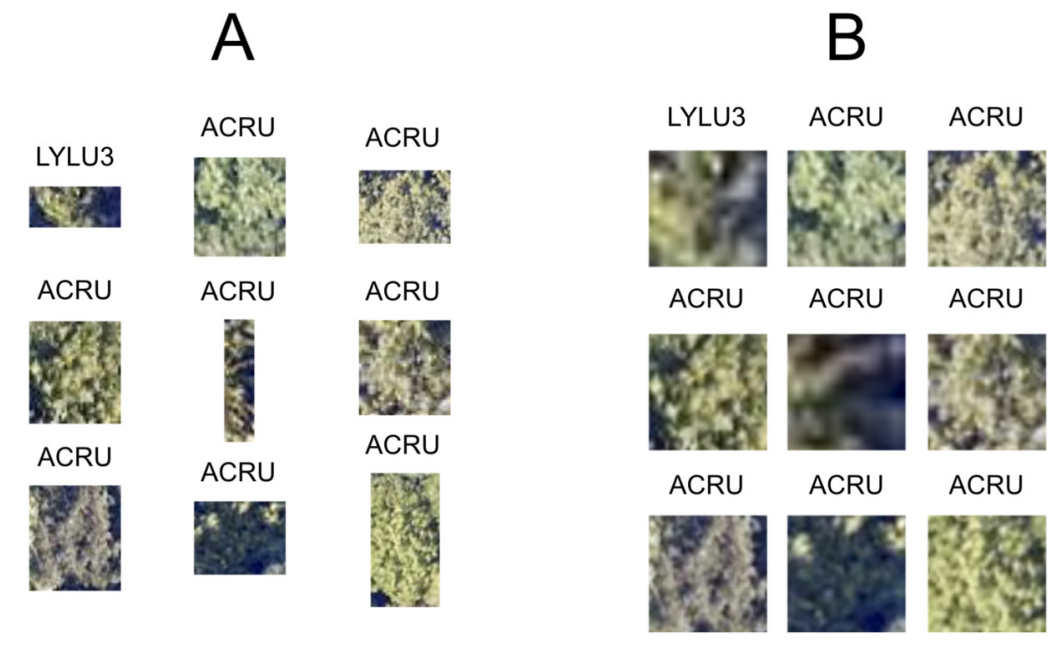

Figure 4. Nine corresponding pairs of RGB image chips, cropped using individual tree crown polygons, with their original crown dimensions (A) and after being resized to $224 \times 224$ pixels (B) to yield consistently shaped inputs for the ResNet classifier. Each image chip is labeled with the taxon identification code that corresponds to each individual plant's scientific name.

that varied in depth including architectures with 18, 34, 50, 101, and 152 layer encoders. We compared these different depth ResNets in terms of the macro F1 score, precision, and recall using our validation subset of the RGB data. The summary of these values is presented in Table 2. 
Table 1. Taxa included in the training data. Each row represents a unique class for the classifier.

\begin{tabular}{|c|c|c|c|}
\hline Taxon code & Scientific name & Common name & Count \\
\hline PIPA2 & Pinus palustris & longleaf pine & 237 \\
\hline QURU & Quercus rubra & northern red oak & 138 \\
\hline ACRU & Acer pensylvanicum & striped maple & 104 \\
\hline QUAL & Quercus alba & white oak & 86 \\
\hline QULA2 & Quercus laevis & turkey oak & 59 \\
\hline QUCO2 & Quercus coccinea & scarlet oak & 39 \\
\hline AMLA & Amelanchier laevis & Allegheny serviceberry & 38 \\
\hline NYSY & Nyssa sylvatica & blackgum & 33 \\
\hline LITU & Liriodendron tulipifera & tuliptree & 16 \\
\hline QUGE2 & Quercus geminata & sand live oak & 15 \\
\hline MAGNO & Magnolia sp. & magnolia & 12 \\
\hline QUMO4 & Quercus montana & chestnut oak & 10 \\
\hline OXYDE & Oxydendrum sp. & sourwood & 9 \\
\hline BETUL & Betula sp. & birch & 6 \\
\hline PINUS & Pinus sp. & pine & 6 \\
\hline PRSE2 & Prunus serotina & black cherry & 6 \\
\hline ACPE & Acer rubrum & red maple & 5 \\
\hline PIEL & Pinus elliottii & slash pine & 4 \\
\hline CAGL8 & Carya glabra & pignut hickory & 3 \\
\hline FAGR & Fagus grandifolia & American beech & 3 \\
\hline PITA & Pinus taeda & loblolly pine & 3 \\
\hline QUHE2 & Quercus hemisphaerica & Darlington oak & 3 \\
\hline ROPS & Robinia pseudoacacia & black locust & 2 \\
\hline TSCA & Tsuga canadensis & eastern hemlock & 2 \\
\hline ACSA3 & Acer saccharum & sugar maple & 1 \\
\hline CATO6 & Carya tomentosa & mockernut hickory & 1 \\
\hline GOLA & Gordonia lasianthus & loblolly bay & 1 \\
\hline LYLU3 & Lyonia lucida & fetterbush lyonia & 1 \\
\hline NYBI & Nyssa biflora & swamp tupelo & 1 \\
\hline QUERC & Quercus sp. & oak & 1 \\
\hline QULA3 & Quercus laurifolia & laurel oak & 1 \\
\hline
\end{tabular}

In a 2-class problem, precision is the proportion of positive predictions which are actually correct, whereas recall is identifies the proportion of actual positive predictions which are correct. F1 score is the harmonic mean of both precision and recall and was an evaluation metric in the competition. To compute the multi-class value of precision, recall, and F1-score, we computed the average across all classes. The Resnet-34 had the highest F1 score, and was used to generate RGB features for the fusion model.

\section{Pseudo-waveform generation from lidar point cloud}

The lidar point cloud contains information on the 3-dimensional structure of tree canopies (Dubayah and Drake, 2000; Kampe et al., 2010b). As the laser travels through the canopy during lidar data collection, energy within the beam's footprint is reflected by the top of the canopy, interactions with sub-canopy elements, and potentially the underlying terrain surface. The returned energy waveform is sampled to produce multiple "returns", points describing the spatial and vertical vegetation structure (Lefsky et al., 2002). The precise 3-dimensional point location is determined by calculating the return time of the reflection from when it was transmitted. Anomalous points can exist, however, and may take the form of points recorded below the ground surface as a result of timing errors in the lidar system due to multiple reflections within the canopy and ground material, or points far above the canopy perhaps due to bird strikes. Often times these points are classified as "Noise" during post-processing, but are not always completely removed. Anomalous points were considered and removed if present by defining valid points as lying between the 1st and 99th percentile of all height values within the point cloud; anomalies were defined as lying outside of those ranges. A comparison showing the point cloud for a single lidar file 
Table 2. Macro F1 score, precision, and recall values for different ResNet convolutional neural network $(\mathrm{CNN})$ architectures that we tested for the red, green, blue (RGB) image classifier.

\begin{tabular}{|c|c|c|c|}
\hline Encoder layers & F1 & Precision & Recall \\
\hline 18 & 0.4282 & 0.3408 & 0.1642 \\
\hline 34 & 0.4698 & 0.1909 & 0.1392 \\
\hline 50 & 0.3463 & 0.2098 & 0.1228 \\
\hline 101 & 0.465 & 0.2916 & 0.1528 \\
\hline 152 & 0.3867 & 0.2635 & 0.1571 \\
\hline
\end{tabular}

before and after removing the height anomalies is shown in Figure 5.

Valid lidar points within each tree crown geometry were used to create a pseudo-waveform for the tree crown which simulates the entire crown's footprint. Muss et al. (2011) have shown this representation of the lidar point cloud to give an accurate representation of vegetation structure as a 1-dimensional signal. We define the pseudo-waveform by calculating the density of points within one-meter height bins ranging from zero meters above ground to the maximum height above ground for any given tree in the training data. This resulted in 39 one-meter height bins ranging from 0 to 40 meters above ground. Bins with no points were given a point density value of zero. This results in a table where each row represents a 1-d structural signal for each tree crown geometry, which are used as additional features in the fusion network for classification. See Figure 6 for example pseudo-waveforms and the corresponding point clouds used to generate them.
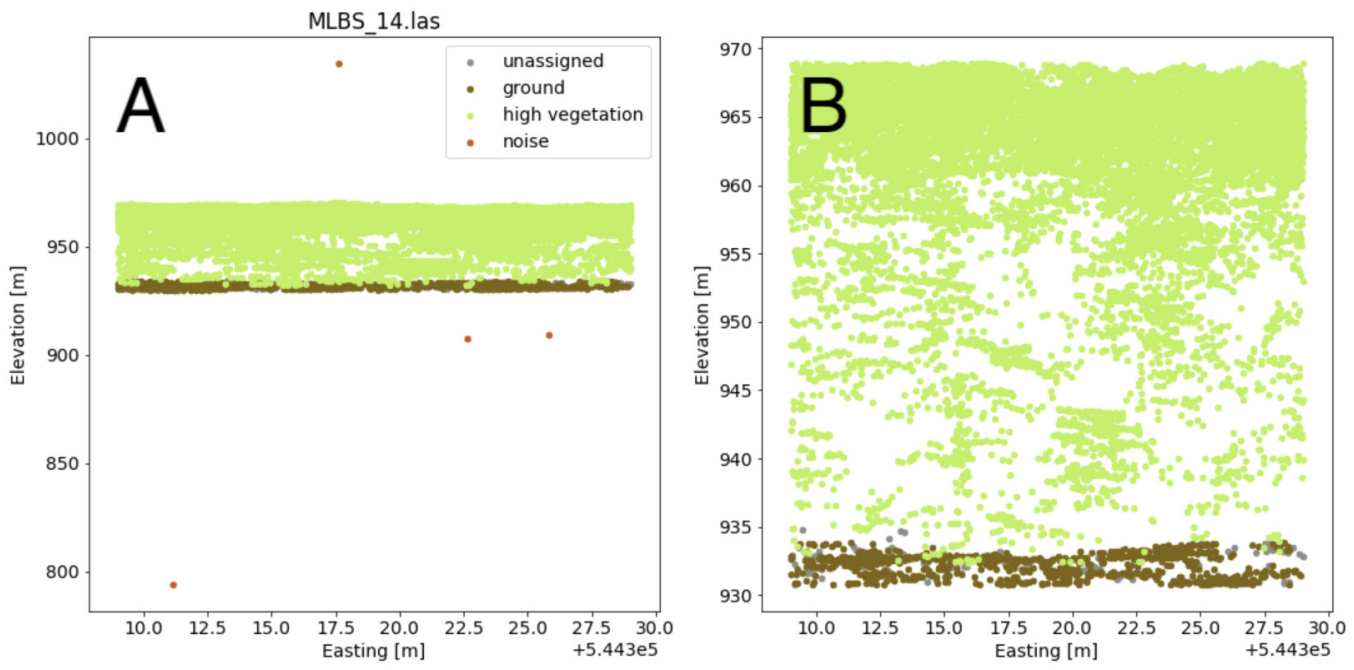

Figure 5. Lidar point cloud showing height anomalies (A) and after height anomalies were removed (B). The point cloud in (B) can be used to generate a pseudo-waveform feature.

\section{The fusion network}

To learn how to combine information from the RGB, hyperspectral, and lidar data, we concatenated the probability vectors from the RGB CNN step, hyperspectral reflectance spectra at the centroid of each tree crown polygon, and lidar pseudo-waveforms into a feature vector that was passed as input to a neural network (also known as a multilayer perceptron), the so-called "fusion network" (Goodfellow et al., 2016). The fusion network was relatively shallow with two hidden layers (size 64 and 32). The input to the fusion network was a feature vector with 440 elements: 31 class probabilities from the RGB ConvNet (one per taxon code), 369 reflectance values from the hyperspectral data (one per wavelength after "bad bands" with high noise due to water absorption were removed), and 40 features from the lidar data (proportions for 39 bins, and the total number of points across all bins). The output of the fusion network was a concatenated vector of taxon probabilities.

In early versions of our model we noticed a tendency to overpredict the most abundant taxa, a problem 

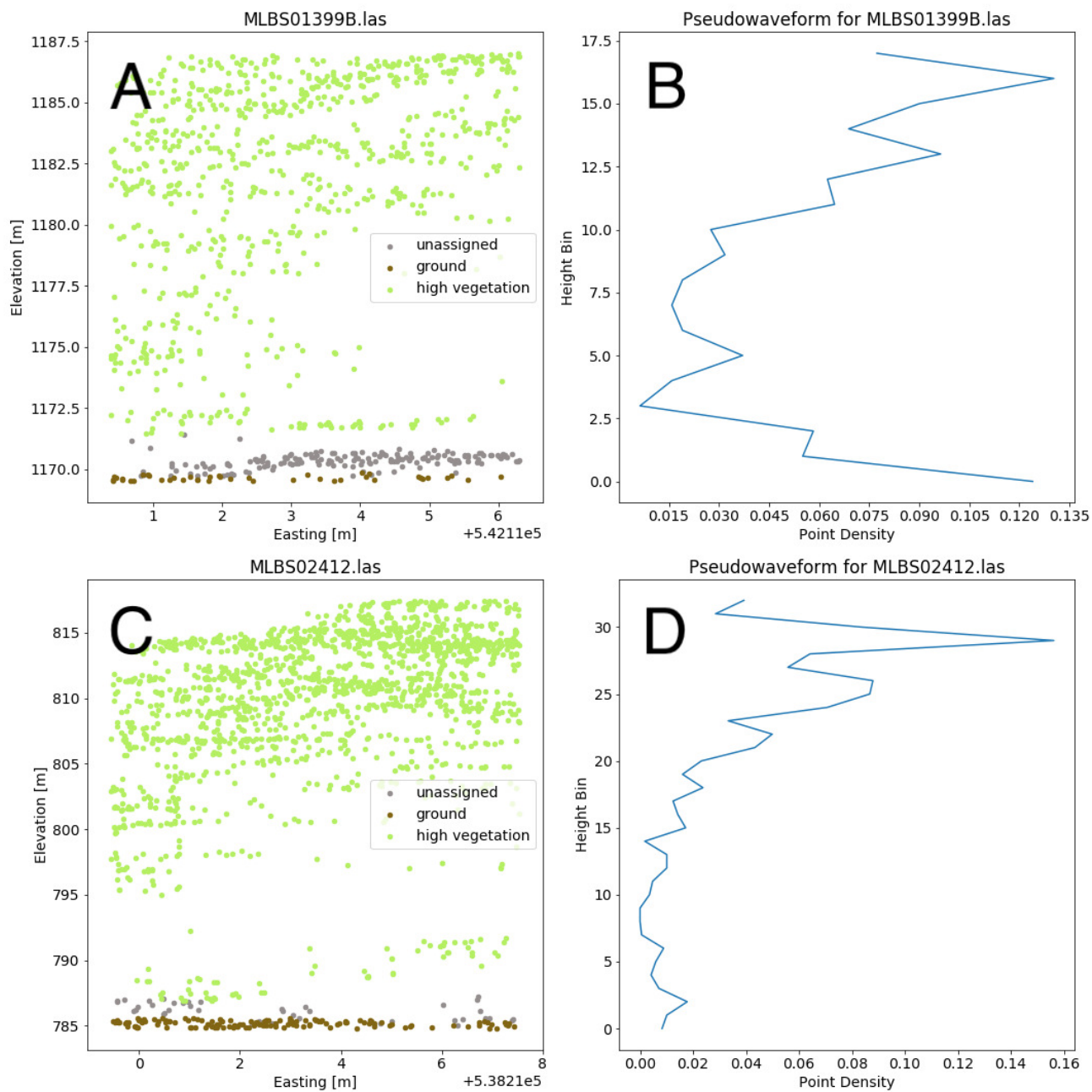

Figure 6. Two examples of different pseudo-waveforms from individual tree crown geometries. Original lidar point clouds $(\mathrm{A}, \mathrm{C})$ and corresponding pseudo-waveforms $(\mathrm{B}, \mathrm{D})$ showing point density at each height bin. Labels are taken from the "indvdID" field from the training data. Note the difference in height values between the two examples.

which we thought might be related to the default cross-entropy loss. We trained the fusion network by minimizing a custom "soft F1" loss function rather than cross-entropy to try to generate predictions that were robust to class imbalance in the training data. Given a classification task with $K$ classes, a length $K$ vector of probabilities $\theta$ and a one-hot-encoded vector $y$ of length $K$, the soft F1 loss can be computed as:

$$
L(\theta)=K^{-1} \sum_{k=1}^{K} 1-\frac{2 \theta_{k} y_{k}}{2 \theta_{k} y_{k}+\theta_{k}\left(1-y_{k}\right)+\left(1-\theta_{k}\right) y_{k}+\varepsilon},
$$

where $\varepsilon$ is a fixed small number (e.g., 1e-7) to prevent division by zero. We used stochastic minibatch gradient descent to minimize the expected soft F1 loss in the training data, using a batch size of 64 examples, averaging loss among examples within each minibatch. The fusion network was trained for 20 epochs using a 1cycle policy, with a maximum learning rate of 1e-2. The number of epochs and the maximum learning rate were chosen based on our $20 \%$ partition of the training data that were set aside as an initial validation set (Smith, 2018).

\section{Post-processing of fusion network output}

To deal with out-of-distribution classes (taxa in the test sites that were not in the training data), we decided to place some probability mass on an "other" class when the model predictions were not confident. If 
the maximum class probability from the fusion network was less than 0.5 , we assigned a probability of 0.5 to the "other" class and renormalized the remaining probabilities so that the entire probability vector summed to one. We chose to use a probability threshold of 0.5 based on qualitative visual inspection of the classification probability histograms.

\section{Implementation}

We processed the RGB and hyperspectral data using GDAL (GDAL/OGR contributors, 2020) and R (R Core Team, 2020). Specifically, we used the neonhs (Joseph and Wasser, 2020) R package to extract hyperspectral reflectance data at the center of each tree crown polygon. We processed the lidar data in Python (Van Rossum and Drake, 2009). As we split the data generation and processing tasks across members of our team, we worked collaboratively and uploaded files to a shared a Google Drive that was readable from Google Colab (Bisong, 2019). We implemented the CNN and fusion network with fastai in Google Colab (Howard and Gugger, 2020). The code that we developed for our methods is openly available on Github (https://github.com/earthlab/idtrees_earthlab) to be freely used and improved upon by the ecological community.

\section{RESULTS}

While initially developing and assessing our methods, we withheld $20 \%$ the training data as an initial validation set, which contained 206 samples spanning all 31 taxon classes. We created a confusion matrix to assess classification accuracy (Fig. 7).

Our initial validation set classification accuracy was 0.51 . The taxa with the most accurate predictions in descending order were PIPA2, ACRU, QUAL, QURU, QULA2, QUCO2, NYSY, and PIEL, many of which were among the most abundant in the training data set (Table 1).

For the final competition evaluation, we applied our classifier to a test data set without knowing the true taxon labels. We submitted a file with our predicted probabilities that each individual plant in the test set belonged to each taxon class, including an unknown class, "other". The IDTReeS competition organizers compared our submitted predictions to the true taxon class labels for each tree crown and provided us with a reduced confusion matrix and a corresponding score report based on true class accuracy. The reduced confusion matrix compares the true and predicted labels for each tree, grouping all out-of-sample taxa into a single class called "other" (Fig. 8). This was done to see the direct match between our predictions of the "other" class with the correct label of "other".

The score report provided based on our predicted taxon labels includes the following metrics calculated using the scikit-learn Python library (Pedregosa et al., 2011): macro average F1 score, weighted average F1 score, and accuracy score from scikit-learn's "classification_report". The Macro Average F1 score considers all predictions from all classes when calculating the F1, whereas the weighted average F1 score considers the relative number of samples per class while computing the F1 score. The accuracy score calculates the global fraction of correct predictions. Our scores for each of these evaluation metrics are summarized in Table 3, with the full set of scores for each species shown in Table 4.

Table 3. Taxon prediction results summarized by competition evaluation metrics.

\begin{tabular}{|c|c|}
\hline Evaluation Metric & Score \\
\hline Macro Average F1 & 0.07 \\
\hline Weighted Average F1 & 0.31 \\
\hline Accuracy & 0.32 \\
\hline Categorical Cross-Entropy & 11.62 \\
\hline
\end{tabular}

\section{DISCUSSION}

Here we presented our plant taxon classification approach that combines a convolutional neural network (CNN) for RGB images with a downstream fusion network that integrates RGB, hyperspectral, and lidar data. Tree species classification accuracy values vary wildly throughout the literature, based on factors such as the number of species being classified and the types of remote sensing systems that captured the data. For instance, a recent review of 101 studies found reported accuracies ranging from less than $60 \%$ 
Tabular model confusion matrix: predicted vs. true label for validation set

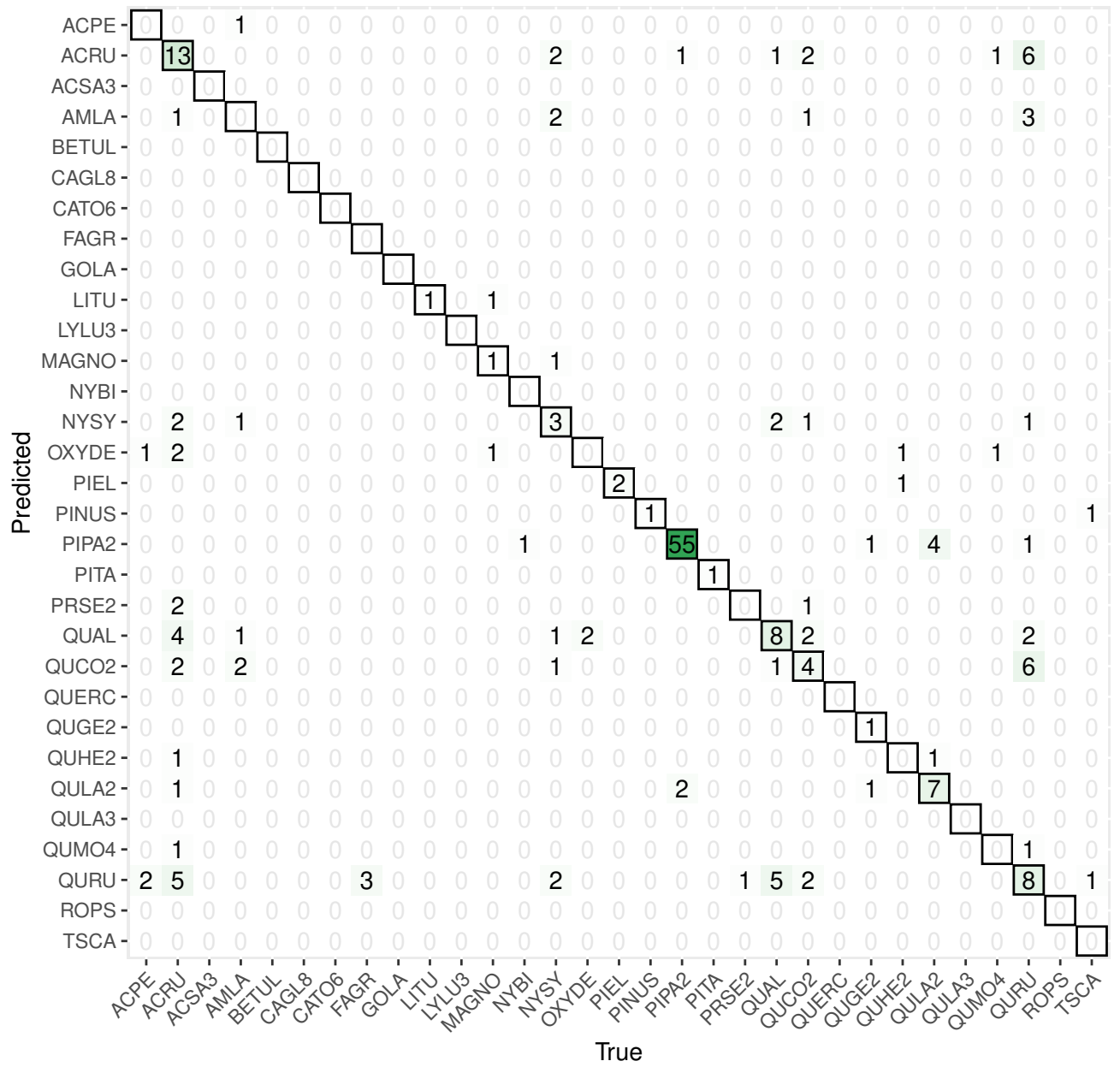

Figure 7. This confusion matrix compares the true and predicted taxon class labels using our tabular classification model. The data used here consist of the $20 \%$ validation subset from the training data. Counts along the diagonal indicate correct predictions.

to nearly $100 \%$ to classify anywhere from a couple to nearly 30 species using combined sensor systems (Fassnacht et al., 2016).

Our classification workflow combined data from all three National Ecological Observatory Network (NEON) airborne remote sensing systems and yielded an overall accuracy of 0.51 for a subset of the training set and 0.32 for the competition test set. The accuracy values that our method achieved are on the low end of the range reported by Fassnacht et al. (2016), although it is worth noting that our method was tasked with classifying 31 species or genera in addition to identifying a series of unknown species in the final competition evaluation, which exceeds the high end of the number of species that the studies classified in the recent review (from a couple to less than 30). For the five participating teams in this IDTReeS competition, the overall classification accuracy values ranged between 0.32 and 0.47 , macro average F1 scores ranged between 0.07 and 0.28 , weighted average F1 scores ranged between 0.31 and 0.45 , and cross entropy scores ranged between 2.5 and 11.62. While our model did well for common classes, poor performance on rare and out-of-distribution classes was a major limitation. The large difference between the macro average F1 score and the weighted average F1 score for the classifier is indicative of the class imbalance and poor classifier performance for rare classes. Table 4 shows the class imbalance present in the test dataset which is reflected in the test dataset confusion matrix, Figure 8. Based on the confusion matrices from the training set (Fig. 7) and test set (Fig. 8), our model struggled to perform as well at the unseen site and unknown taxon classes. We obtained an overall accuracy of 0.51 
Confusion matrix: true vs. predicted label for competition test set

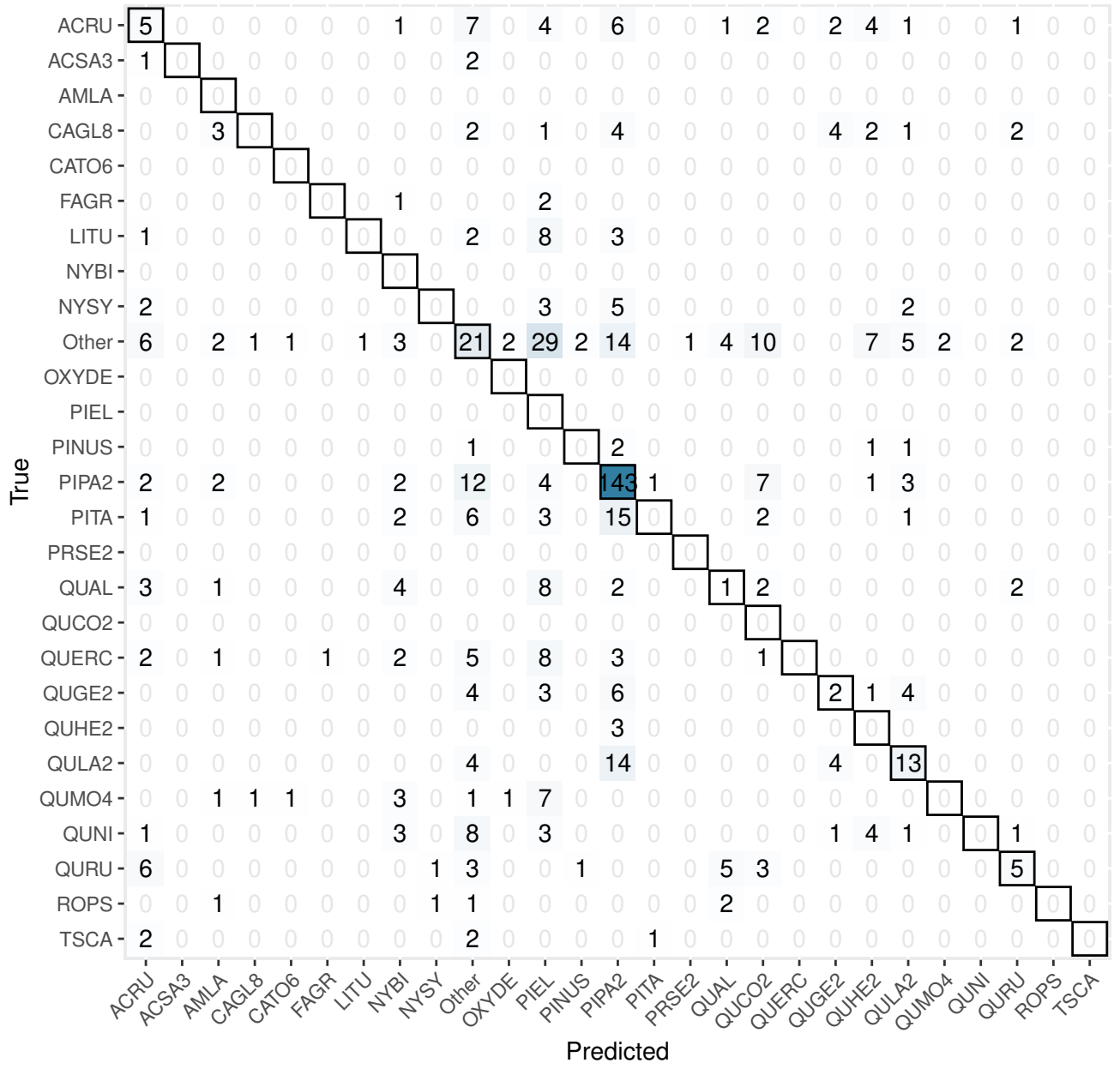

Figure 8. This confusion matrix compares the true and predicted taxon class labels using our tabular classification model. The data used here consist of the test set for the final competition evaluation. Counts along the diagonal indicate correct predictions.

when predicting the taxon labels in our 20\% withheld from training, which was higher than the overall accuracy of 0.32 reported for the test set, which might be indicative of overfitting on the validation data.

Aside from overfitting, poor performance on out-of-distribution data could be due to dataset shifts or differences at the third NEON site. For instance, we found image artifacts such as distortion or the presence of shadows due to illumination conditions are variable across plots and NEON sites, which are visible in Figure 3. Lots of distortion was visible in images from the OSBS site, likely an effect of wind during the data collection flight. Note that the presence and appearance of this distortion is not consistent across the images. These artifacts in addition to the highly variable individual tree crown polygons, which we transformed to be squares of uniform size, likely challenged the RGB portion of our classification approach. We discussed the possibility of filtering small or oddly shaped crowns (i.e. one pixel wide by six pixels tall) since these shapes may be due to occlusion by neighboring crowns on a per-case basis, and may not necessarily be representative of that taxon's typical crown dimensions. However, without doing more in-depth analysis about which shapes or dimensions to filter, we kept all individual tree crown shapes in the data set for our analysis.

Poor performance for out-of-distribution data could also be attributed to uncertainty calibration for the "other" class. Our approach to deal with these unknowns was to use a 0.5 certainty threshold to label an individual as "other". We correctly identified 21 of 113 trees with true labels of "other", which amounts to $22 \%$ of them. As described in our methods section, our decision to use this 0.5 threshold was based on 
Table 4. Full report of competition classification evaluation metrics. These test set results include the classifier total accuracy, Macro F1 score, and weighted average F1 score in bold.

\begin{tabular}{|l|l|l|l|l|}
\hline & precision & recall & f1-score & support \\
\hline ACRU & 0.15625 & 0.147059 & 0.151515 & 34 \\
\hline ACSA3 & 0 & 0 & 0 & 3 \\
\hline AMLA & 0 & 0 & 0 & 0 \\
\hline CAGL8 & 0 & 0 & 0 & 19 \\
\hline CATO6 & 0 & 0 & 0 & 0 \\
\hline FAGR & 0 & 0 & 0 & 3 \\
\hline LITU & 0 & 0 & 0 & 14 \\
\hline NYBI & 0 & 0 & 0 & 0 \\
\hline NYSY & 0 & 0 & 0 & 12 \\
\hline OXYDE & 0 & 0 & 0 & 0 \\
\hline Other & 0.259259 & 0.185841 & 0.216495 & 113 \\
\hline PIEL & 0 & 0 & 0 & 0 \\
\hline PINUS & 0 & 0 & 0 & 5 \\
\hline PIPA2 & 0.65 & 0.80791 & 0.720403 & 177 \\
\hline PITA & 0 & 0 & 0 & 30 \\
\hline PRSE2 & 0 & 0 & 0 & 0 \\
\hline QUAL & 0.076923 & 0.043478 & 0.055556 & 23 \\
\hline QUCO2 & 0 & 0 & 0 & 0 \\
\hline QUERC & 0 & 0 & 0 & 23 \\
\hline QUGE2 & 0.153846 & 0.1 & 0.121212 & 20 \\
\hline QUHE2 & 0 & 0 & 0 & 3 \\
\hline QULA2 & 0.40625 & 0.371429 & 0.38806 & 35 \\
\hline QUMO4 & 0 & 0 & 0 & 15 \\
\hline QUNI & 0 & 0 & 0 & 22 \\
\hline QURU & 0.384615 & 0.208333 & 0.27027 & 24 \\
\hline ROPS & 0 & 0 & 0 & 5 \\
\hline TSCA & 0 & 0 & 0 & 5 \\
\hline accuracy & $\mathbf{0 . 3 2 4 7 8 6}$ & $\mathbf{0 . 3 2 4 7 8 6}$ & $\mathbf{0 . 3 2 4 7 8 6}$ & $\mathbf{0 . 3 2 4 7 8 6}$ \\
\hline macro avg & 0.077302 & 0.069039 & $\mathbf{0 . 0 7 1 2 4 1}$ & 585 \\
\hline weighted avg & 0.304196 & 0.324786 & $\mathbf{0 . 3 0 9 2 2 6}$ & $\mathbf{5 8 5}$ \\
\hline
\end{tabular}

the distribution of probabilities observed during training. Further tuning or increasing this threshold may lead to better identification of unknown taxon classes in the future.

We spent some time brainstorming different approaches to handle out of distribution classes (taxa present in the test set that were absent in the training data). Our final solution to this (ad hoc "other" class predictions) was a much simpler version compared to some of the ideas that we had. Most elaborate among these abandoned ideas was to use $\mathrm{K}$-fold cross-validation to iteratively generate $\mathrm{K}$ train/validation splits of the training data, some of which would result in some taxa being only represented in the validation data. Our thought was to try to build a model that was well-calibrated based on this cross-validation, i.e., a model that was able to predict "other" when presented with a taxon that was not represented in the training data.

Related to predictive features to train the classifier, we investigated texture measures from the RGB data as a potential set of features to use as inputs for classification. Preliminary analysis on Haralick texture features (Haralick et al., 1973), calculated from each tree's gray-level co-occurrence matrix, did not prove separable at the taxon level when considering the training data. A principal components transform (Rodarmel and Shan, 2002) was applied to the texture feature space, but the transformed axes did not prove separable, either. We also explored dimensionality reduction methods directly with the hyperspectral data, which are commonly used to summarize data from hundreds of highly correlated hyperspectral bands into fewer bands (Fassnacht et al., 2016; Maschler et al., 2018). Another approach to perform dimensionality reduction would be to use an auto-encoder (Wang et al., 2016). Including 
additional descriptive features as a result of dimensionality reduction methods like principal component analysis, spectral indices, or targeted feature selection such as specific spectral bands may improve future classifier efforts. Additional ideas for improving classifier performance in the data preprocessing steps include identifying and removing (or utilizing) non-vegetation and shadow pixels (Mostafa, 2017), which are especially visible in the high spatial resolution RGB images (Fig. 3).

We made use of the lidar point cloud data by resampling the points into pseudo-waveforms, which allowed us to incorporate information about point density at different heights within the canopy. Future classification methods may benefit from incorporating additional point cloud-derived metrics, such as modeling the shape of the crown, distances between first and last returns, as well as intensity information, although this may require data with a higher point density (Korpela et al., 2010). The only competition dataset that we did not incorporate into our classifier was the rasterized lidar-derived canopy height model (CHM) (Goulden and Scholl, 2019). The CHM data was at 1 meter per pixel resolution and we felt that it did not provide enough information relative to the other datasets, particularly its cohort of the feature-rich 1 meter hyperspectral imagery. Thus, we made an executive decision to not include the data as the boundaries of the tree crowns as observed in the RGB data (Fig. 3) were much too coarse to justify using the CHM as a means by which to crop any of the other data. However, with higher resolution CHM data or larger crown geometries, we foresee being able to directly use the CHM information about crown geometry to generate better data subsets and extractions for individual trees (Scholl et al., 2020).

Early on in the competition, we discussed the merits of a one- versus two-stage approach for data integration. While we settled on a two-stage approach (CNN to fusion network), a one-stage approach might have been a viable option. In a one-stage approach, we would embed the CNN within the fusion network, and instead of passing the output to a downstream model, we would concatenate the feature vector generated from the convnet with the vector valued features in the fusion network to obtain a model that is end-to-end differentiable. It was not clear that this would result in a better model, but it was clear it would require considerably more effort.

\section{CONCLUSIONS}

The IDTreeS 2020 plant classification challenge openly invited teams to create and compare their methods using open-source NEON data. In this paper, we presented the methods and results of the team called Jeepers Treepers. We used a two-stage deep learning fusion network approach to combine features from $\mathrm{RGB}$, hyperspectral, and lidar point cloud data to classify taxa at an unseen site featuring unknown species. Creating classification methods that are transferable and generalizeable is no easy task, which made it an interesting topic for this data competition. Overall, we believe that further processing and filtering the RGB images (such as calculating texture metrics and manually removing images containing notable image artifacts or non-vegetation pixels), refining the logic for identifying unknown taxa (when assigning individuals to the "other" class), further addressing the taxon imbalance in the training data set, and incorporating greater data volume and features (such as additional lidar point cloud metrics based on point height and intensity) would improve our classifier's performance. We see value in the open data-driven competition format to accelerate methods development in the computational ecology field, and encourage others to participate in the future.

\section{ACKNOWLEDGMENTS}

We would like to thank the members of the Earth Lab Deep Learning meet up group for brainstorming about our methods during Spring 2020. We also appreciate the IDTReeS competition organizers' flexibility and responsiveness during the unprecedented year of 2020.

\section{REFERENCES}

Abdollahnejad, A. and Panagiotidis, D. (2020). Tree species classification and health status assessment for a mixed broadleaf-conifer forest with uas multispectral imaging. Remote Sensing, 12(22):3722.

Anderson, J. E., Plourde, L. C., Martin, M. E., Braswell, B. H., Smith, M.-L., Dubayah, R. O., Hofton, M. A., and Blair, J. B. (2008). Integrating waveform lidar with hyperspectral imagery for inventory of a northern temperate forest. Remote Sensing of Environment, 112(4):1856-1870.

Asner, G. P., Jones, M. O., Martin, R. E., Knapp, D. E., and Hughes, R. F. (2008). Remote sensing of native and invasive species in hawaiian forests. Remote Sensing of Environment, 112(5):1912-1926. 
Asner, G. P., Knapp, D. E., Boardman, J., Green, R. O., Kennedy-Bowdoin, T., Eastwood, M., Martin, R. E., Anderson, C., and Field, C. B. (2012). Carnegie airborne observatory-2: Increasing science data dimensionality via high-fidelity multi-sensor fusion. Remote Sensing of Environment, 124:454-465.

Ballanti, L., Blesius, L., Hines, E., and Kruse, B. (2016). Tree species classification using hyperspectral imagery: A comparison of two classifiers. Remote Sensing, 8(6):445.

Bisong, E. (2019). Google colaboratory. In Building Machine Learning and Deep Learning Models on Google Cloud Platform, pages 59-64. Springer.

Brodrick, P. G., Davies, A. B., and Asner, G. P. (2019). Uncovering ecological patterns with convolutional neural networks. Trends in ecology \& evolution, 34(8):734-745.

Carpenter, J. (2011). May the best analyst win.

Dalponte, M., Ørka, H. O., Gobakken, T., Gianelle, D., and Næsset, E. (2012). Tree species classification in boreal forests with hyperspectral data. IEEE Transactions on Geoscience and Remote Sensing, 51(5):2632-2645.

Deng, J., Dong, W., Socher, R., Li, L.-J., Li, K., and Fei-Fei, L. (2009). Imagenet: A large-scale hierarchical image database. In 2009 IEEE conference on computer vision and pattern recognition, pages 248-255. Ieee.

Diaz, J., St Denis, L., Joseph, M. B., Solvik, K., and Balch, J. K. (2020). Classifying twitter users for disaster response: A highly multimodal or simple approach? In Proceedings of the Information Systems for Crisis Response and Management Conference (ISCRAM 2020).

Dubayah, R. O. and Drake, J. B. (2000). Lidar remote sensing for forestry. Journal of Forestry, 98(6):4446.

Fassnacht, F. E., Latifi, H., Stereńczak, K., Modzelewska, A., Lefsky, M., Waser, L. T., Straub, C., and Ghosh, A. (2016). Review of studies on tree species classification from remotely sensed data. Remote Sensing of Environment, 186:64-87.

Fricker, G. A., Ventura, J. D., Wolf, J. A., North, M. P., Davis, F. W., and Franklin, J. (2019). A convolutional neural network classifier identifies tree species in mixed-conifer forest from hyperspectral imagery. Remote Sensing, 11(19):2326.

Gallery, W., Goulden, T., Leisso, N., and Krause, K. (2015). Neon aop digital camera image orthorectification algorithm theoretical basis document (atbd) neon.doc.001211. The National Ecological Observatory Network.

GDAL/OGR contributors (2020). GDAL/OGR Geospatial Data Abstraction software Library. Open Source Geospatial Foundation.

Gini, R., Sona, G., Ronchetti, G., Passoni, D., and Pinto, L. (2018). Improving tree species classification using uas multispectral images and texture measures. ISPRS International Journal of Geo-Information, $7(8): 315$.

Goodfellow, I., Bengio, Y., Courville, A., and Bengio, Y. (2016). Deep learning, volume 1. MIT press Cambridge.

Goulden, T. and Scholl, V. (2019). Neon ecosystem structure algorithm theoretical basis document (atbd) neon.doc.002387. The National Ecological Observatory Network.

Graves, S. and Marconi, S. (2020). Idtrees 2020 competition data (version 4). This data is supported by the National Science Foundation through grant 1926542 and by the Gordon and Betty Moore Foundation's Data-Driven Discovery Initiative through grant GBMF4563 to E.P. White, and the NSF Dimension of Biodiversity program grant (DEB-1442280) and USDA/NIFA McIntire-Stennis program (FLA-FOR-005470).

Haralick, R. M., Shanmugam, K., and Dinstein, I. H. (1973). Textural features for image classification. IEEE Transactions on systems, man, and cybernetics, (6):610-621.

He, K., Zhang, X., Ren, S., and Sun, J. (2016). Deep residual learning for image recognition. In Proceedings of the IEEE conference on computer vision and pattern recognition, pages 770-778.

He, K. S., Rocchini, D., Neteler, M., and Nagendra, H. (2011). Benefits of hyperspectral remote sensing for tracking plant invasions. Diversity and Distributions, 17(3):381-392.

Heinzel, J. and Koch, B. (2011). Exploring full-waveform lidar parameters for tree species classification. International Journal of Applied Earth Observation and Geoinformation, 13(1):152-160.

Howard, J. and Gugger, S. (2020). Fastai: A layered api for deep learning. Information, 11(2):108.

Johnson, B. R., Kuester, M. A., Kampe, T. U., and Keller, M. (2010). National ecological observatory network (neon) airborne remote measurements of vegetation canopy biochemistry and structure. In 
2010 IEEE International Geoscience and Remote Sensing Symposium, pages 2079-2082. IEEE.

Joseph, M. and Wasser, L. (2020). neonhs: Work with NEON AOP hyperspectral data. R package version 0.0.9999.

Jucker, T., Caspersen, J., Chave, J., Antin, C., Barbier, N., Bongers, F., Dalponte, M., van Ewijk, K. Y., Forrester, D. I., Haeni, M., et al. (2017). Allometric equations for integrating remote sensing imagery into forest monitoring programmes. Global change biology, 23(1):177-190.

Kampe, T. U., Asner, G. P., Green, R. O., Eastwood, M., Johnson, B. R., and Kuester, M. (2010a). Advances in airborne remote sensing of ecosystem processes and properties: toward high-quality measurement on a global scale. In Remote Sensing and Modeling of Ecosystems for Sustainability VII, volume 7809, page 78090J. International Society for Optics and Photonics.

Kampe, T. U., Johnson, B. R., Kuester, M. A., and Keller, M. (2010b). Neon: the first continental-scale ecological observatory with airborne remote sensing of vegetation canopy biochemistry and structure. Journal of Applied Remote Sensing, 4(1):043510.

Karpowicz, B. and Kampe, T. (2015). Neon imaging specttrometer radiance to reflectance algorithm theoretical basis document (atbd) neon.doc.001288. The National Ecological Observatory Network.

Keller, M., Schimel, D. S., Hargrove, W. W., and Hoffman, F. M. (2008). A continental strategy for the national ecological observatory network. The Ecological Society of America: 282-284.

Kerr, J. T. and Ostrovsky, M. (2003). From space to species: ecological applications for remote sensing. Trends in ecology \& evolution, 18(6):299-305.

Koenig, K. and Höfle, B. (2016). Full-waveform airborne laser scanning in vegetation studies-a review of point cloud and waveform features for tree species classification. Forests, 7(9):198.

Korpela, I., Ørka, H. O., Maltamo, M., Tokola, T., and Hyyppä, J. (2010). Tree species classification using airborne lidar-effects of stand and tree parameters, downsizing of training set, intensity normalization, and sensor type. Silva Fennica, 44(2):319-339.

Krause, K. and Goulden, T. (2015). Neon level 0 to level 1 discrete-return lidar algorithm theoretical basis document (atbd) neon.doc.001292. The National Ecological Observatory Network.

Kulakowski, D., Veblen, T. T., and Bebi, P. (2003). Effects of fire and spruce beetle outbreak legacies on the disturbance regime of a subalpine forest in colorado. Journal of Biogeography, 30(9):1445-1456.

LeCun, Y., Bengio, Y., and Hinton, G. (2015). Deep learning. nature, 521(7553):436-444.

Lefsky, M. A., Cohen, W. B., Parker, G. G., and Harding, D. J. (2002). Lidar remote sensing for ecosystem studies: Lidar, an emerging remote sensing technology that directly measures the three-dimensional distribution of plant canopies, can accurately estimate vegetation structural attributes and should be of particular interest to forest, landscape, and global ecologists. BioScience, 52(1):19-30.

Lucash, M. S., Scheller, R. M., Sturtevant, B. R., Gustafson, E. J., Kretchun, A. M., and Foster, J. R. (2018). More than the sum of its parts: how disturbance interactions shape forest dynamics under climate change. Ecosphere, 9(6):e02293.

Ma, L., Liu, Y., Zhang, X., Ye, Y., Yin, G., and Johnson, B. A. (2019). Deep learning in remote sensing applications: A meta-analysis and review. ISPRS journal of photogrammetry and remote sensing, 152:166-177.

Marconi, S., Graves, S. J., Gong, D., Nia, M. S., Le Bras, M., Dorr, B. J., Fontana, P., Gearhart, J., Greenberg, C., and Harris, D. J. (2019). A data science challenge for converting airborne remote sensing data into ecological information. PeerJ, 6:e5843.

Maschler, J., Atzberger, C., and Immitzer, M. (2018). Individual tree crown segmentation and classification of 13 tree species using airborne hyperspectral data. Remote Sensing, 10(8):1218.

Moon, J. B., Dewitt, T. H., Errend, M. N., Bruins, R. J., Kentula, M. E., Chamberlain, S. J., Fennessy, M. S., and Naithani, K. J. (2017). Model application niche analysis: assessing the transferability and generalizability of ecological models. Ecosphere, 8(10):e01974.

Mostafa, Y. (2017). A review on various shadow detection and compensation techniques in remote sensing images. Canadian journal of remote sensing, 43(6):545-562.

Muss, J., Mladenoff, D., and Townsend, P. (2011). A pseudo-waveform technique to assess forest structure using discrete lidar data. Remote Sensing of Environment, 115:824-835.

Nagendra, H. (2001). Using remote sensing to assess biodiversity. International journal of remote sensing, 22(12):2377-2400.

NEON, N. E. O. N. (2020). Data Products: DP1.30010.001, DP1.30003.001, DP1.10098.001, DP3.30006.003. Provisional data downloaded from http://data.neonscience.org on 19 May 2019. 
Battelle, Boulder, CO, USA.

Ngiam, J., Khosla, A., Kim, M., Nam, J., Lee, H., and Ng, A. Y. (2011). Multimodal deep learning. In ICML.

Onishi, M. and Ise, T. (2021). Explainable identification and mapping of trees using uav rgb image and deep learning. Scientific reports, 11(1):1-15.

Pedregosa, F., Varoquaux, G., Gramfort, A., Michel, V., Thirion, B., Grisel, O., Blondel, M., Prettenhofer, P., Weiss, R., Dubourg, V., Vanderplas, J., Passos, A., Cournapeau, D., Brucher, M., Perrot, M., and Duchesnay, E. (2011). Scikit-learn: Machine learning in Python. Journal of Machine Learning Research, 12:2825-2830.

R Core Team (2020). R: A Language and Environment for Statistical Computing. R Foundation for Statistical Computing, Vienna, Austria.

Rodarmel, C. and Shan, J. (2002). Principal component analysis for hyperspectral image classification. Surveying and Land Information Science, 62(2):115-122.

Schimel, D., Pavlick, R., Fisher, J. B., Asner, G. P., Saatchi, S., Townsend, P., Miller, C., Frankenberg, C., Hibbard, K., and Cox, P. (2015). Observing terrestrial ecosystems and the carbon cycle from space. Global Change Biology, 21(5):1762-1776.

Scholl, V. M., Cattau, M. E., Joseph, M. B., and Balch, J. K. (2020). Integrating national ecological observatory network (neon) airborne remote sensing and in-situ data for optimal tree species classification. Remote Sensing, 12(9):1414.

Senf, C., Seidl, R., and Hostert, P. (2017). Remote sensing of forest insect disturbances: Current state and future directions. International journal of applied earth observation and geoinformation, 60:49-60.

Smith, L. N. (2018). A disciplined approach to neural network hyper-parameters: Part 1-learning rate, batch size, momentum, and weight decay. arXiv preprint arXiv:1803.09820.

Thorpe, A. S., Barnett, D. T., Elmendorf, S. C., Hinckley, E.-L. S., Hoekman, D., Jones, K. D., LeVan, K. E., Meier, C. L., Stanish, L. F., and Thibault, K. M. (2016). Introduction to the sampling designs of the $\mathrm{n}$ ational e cological o bservatory $\mathrm{n}$ etwork t errestrial o bservation s ystem. Ecosphere, 7(12):e01627.

Torabzadeh, H., Morsdorf, F., and Schaepman, M. E. (2014). Fusion of imaging spectroscopy and airborne laser scanning data for characterization of forest ecosystems-a review. ISPRS Journal of Photogrammetry and Remote Sensing, 97:25-35.

Tusa, E., Laybros, A., Monnet, J.-M., Dalla Mura, M., Barré, J.-B., Vincent, G., Dalponte, M., Feret, J.-B., and Chanussot, J. (2020). Fusion of hyperspectral imaging and lidar for forest monitoring. In Data Handling in Science and Technology, volume 32, pages 281-303. Elsevier.

Van Rossum, G. and Drake, F. L. (2009). Python 3 Reference Manual. CreateSpace, Scotts Valley, CA. Wang, K., Franklin, S. E., Guo, X., and Cattet, M. (2010). Remote sensing of ecology, biodiversity and conservation: a review from the perspective of remote sensing specialists. Sensors, 10(11):9647-9667.

Wang, Y., Yao, H., and Zhao, S. (2016). Auto-encoder based dimensionality reduction. Neurocomputing, 184:232-242.

White, J. C., Coops, N. C., Wulder, M. A., Vastaranta, M., Hilker, T., and Tompalski, P. (2016). Remote sensing technologies for enhancing forest inventories: A review. Canadian Journal of Remote Sensing, 42(5):619-641.

Wu, J., Jones, B., Li, H., and Loucks, O. L. (2006). Scaling and uncertainty analysis in ecology. Springer. Zhang, C., Xia, K., Feng, H., Yang, Y., and Du, X. (2020). Tree species classification using deep learning and rgb optical images obtained by an unmanned aerial vehicle. Journal of Forestry Research, pages $1-10$.

Zhu, X. X., Tuia, D., Mou, L., Xia, G.-S., Zhang, L., Xu, F., and Fraundorfer, F. (2017). Deep learning in remote sensing: A comprehensive review and list of resources. IEEE Geoscience and Remote Sensing Magazine, 5(4):8-36. 